\title{
Design and Development of Analysis Tools of Uniform Angle Index
}

\author{
H.Y. ZHAO, J. ZHANG*, M.X. ZHANG \& H.B. ZHENG \\ Forest Fire Department of Nanjing Forest Police College, Nanjing, JiangSu Province 210023, P.R. China
}

*Corresponding Author: zhy62391351@163.com

\begin{abstract}
Analysis Tools of Uniform Angle Index is of great importance to structure-based forest management, however, few convenient tools were developed for practical use. In order to facilitate calculating Uniform Angle Index of each tree and the mean value of uniform angle index of stand in forest, a tool was developed. Firstly, the distances from four closest neighboring trees to reference tree were separately computed with the formula of the distance. Secondly, four angles between the lines passing through the reference tree and other trees and $\mathrm{x}$-axis were counted with the function Atan2 ( ) and the standard angles were determined in line with subtraction of the angles $(\theta)$. Thirdly, uniform angle index of each tree and the mean value of uniform angle index of stand in forest were computed. Testing results show that the tool can precisely calculate uniform angle index.
\end{abstract}

KEYWORD: Structure-based forest management; Uniform angle index; Excel VBA

\section{INTRODUCTION}

Forests can provide multiple services, including economic benefit, ecological benefit and social benefit. The global value of forest ecosystem goods and services has been estimated at $\$ 4.7$ trillion annually (Hui \& Klaus von Gadow, et al. 2007). The functions of a system are determined to a large extent by its structure. The spatial structure refers to the spatial relationships among different species in a forest community and the spatial arrangement of their attributes. The spatial pattern of trees is the integration of biological properties of the population, intraspecific and interspecific relationship and environmental conditions (Hui \& Klaus von Gadow, et al. 2004). Author \& Author 1987).

So far, more and more people have pay attention to trees' spatial pattern and more good methods have been presented. The common methods of analyzing trees' spatial pattern contain geo-statistics, nearestneighbor method and the function of Ripley'S K(d). In 1999, Hui et al. proposed a structure parameter for describing distribution of forest tree positionuniform angle index and then have developed a forest management approach based on uniform angle index and other structure parameters (Hui \& Klaus 1999).
Generally, the data which were collected from field survey were stored in Excel workbooks and Excel workbook itself has very powerful statistical analysis and charting function. Excel VBA programming language embedded in Excel can manipulate directly workbooks of Microsoft Excel (Zhao \& Kang, et al. 2013). Therefore, it is feasible way to develop softwares with Excel VBA. VBA has been mostly preferred for systems development in recent years. Up to now, some DBMISs (data base management information systems), GISs (geographic information systems) (Zhou \& Li, et al. 2006), DSSs (decision support systems) (Zhao \& Lu, et al. 2011) and special software in regression analysis and charting (Tang \& Zhao, et al. 2011; Zhao \& Kang, et al. 2011; Zhao \& Kang, et al. 2011; Wang 2009) have been developed.

The goal of this paper was to design and develop a tool that can calculate uniform Angle Index with VBA.

\section{DESIGN OF ANALYSIS TOOLS OF UNIFORM ANGLE INDEX}

\subsection{Basic Idea}

Four closest neighboring trees of a reference tree could be chosen in a stand. The included angle 
between any two closest neighboring trees with the reference tree consist of a larger angle $(\beta)$ and a smaller angle $(\alpha)$ and $\alpha$ and $\beta$ equal to 360 (Figure1). ais usually described as the standard angles. Uniform angle index $\left(\mathrm{W}_{\mathrm{i}}\right)$ is a ratio of the number of $\alpha\left(\leq 72^{\circ}\right)$ to four. Wi can be expressed by the follow expression:

$$
\begin{aligned}
& W_{i}=\frac{1}{4} \sum_{j=1}^{4} Z_{i j} \\
& Z_{i j}=\left\{\begin{array}{c}
1, a \leq 72 \\
0, o r
\end{array}\right.
\end{aligned}
$$

When $\mathrm{W}_{\mathrm{ij}}=0$, four closest neighboring trees around the reference tree are very unevenly distributed. When $\mathrm{W}_{\mathrm{ij}}=1$, four closest neighboring trees around the reference tree are extremely nonuniform distributed.

In order to develop a tool to calculate uniform angle index, two key points need be solved:

1. Four closest neighboring trees of a reference tree should be found out. According to the formula of the distance, the distances between the reference tree and the neighboring trees in a stand are separately calculated. Four closest neighboring trees are chosen by utilizing the function of minimum value transmission;

2. The standard angles are acquired. Firstly, the angles between the lines passing through the reference tree and each neighboring tree and $\mathrm{x}$-axis are computed with the function Atan2 ( ). Moreover, the angles can be used to sort an array from lowest to highest with the function of minimum value transmission. Finally, the angles $\alpha$ are acquired by subtraction of the angles $(\theta)$ between the lines and $\mathrm{x}$ axis (Figure 2) for example, $\alpha_{1}=\theta_{2}-\theta_{1}$.

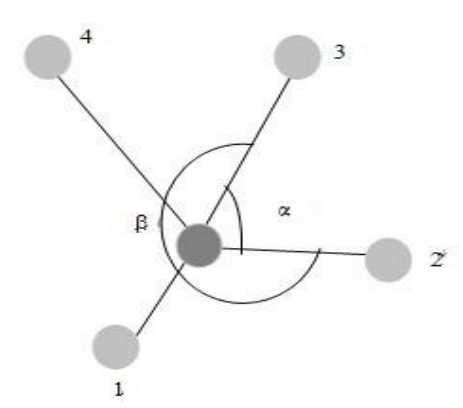

Figure1. Schematic plot of the included angle between any two closest neighboring trees with the reference tree

\subsection{Operation flow of Analysis Tools of Uniform Angle Index}

The distances from four closest neighboring trees to reference tree were separately computed with the formula of the distance. Four closest distances are chosen by utilizing the function of minimum value transmission. Moreover, four angles between the lines passing through the reference tree and other trees and $\mathrm{x}$-axis were counted with the function Atan2 ( ) (Figure6a) and can be used to sort an array from lowest to highest with the function of minimum value transmission (Figure6a). Subsequently, the standard angles were chosen in line with subtraction of the angles $(\theta)$ between the lines and $\mathrm{x}$-axis. The number of $\alpha$ that are greater than 72 degree were counted and $\mathrm{Wi}$ of each reference tree was acquired according to the formula of uniform angle index (Figure6c).

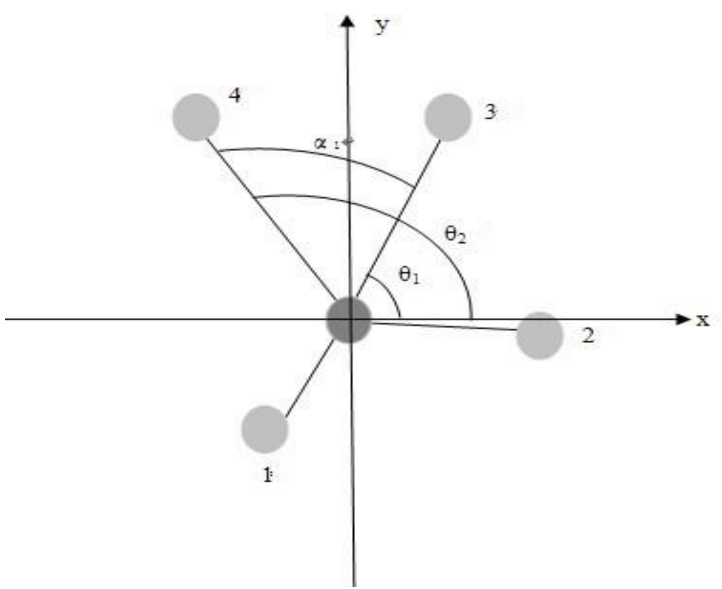

Figure2. Schematic plot of the standard angle acquired by subtraction of the angles $(\theta)$

\subsection{Development of Analysis Tools of Uniform Angle Index}

Generally, when systems are developed by using VBA, fields and format of sheets and main modules should be separately designed in the framework of the systems flow charts. Sheets are mainly used for storing initial data and processed data. Modules are mainly engaged to implement the all functions of the systems or tools.

Analysis Tools of Uniform Angle Index contained two EXCEL sheet, toolbars and abundant program codes embedded in Excel workbook. Toolbars in EXCEL are convenient for computing uniform angle index. Excel sheets were used as database, where data were processed and stored.

\subsubsection{The design of sheets}

Operating environment of the Analysis Tools of Uniform Angle Index is EXCEL. There were two sheets: "Primitive data" and "Mean value of UAI" (Figure5). The two sheets were used for storing initial data and processed data, respectively. The sheet of "Primitive data" insist of four fields: "Tree 
Number"," Tree species"," coordinate(x or y)"," Uniform Angle Index". Data of the first three fields were collected by foresters, and the last one was computed by related module of the tool. The sheet of "Mean value of UAI" was used to store the mean value of uniform angle index of stand (Figure4).

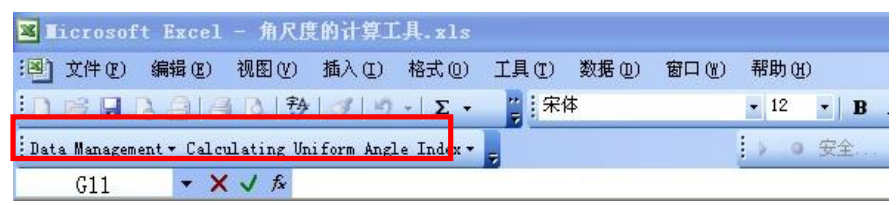

Figure3. User interface of analysis tools of uniform angle index

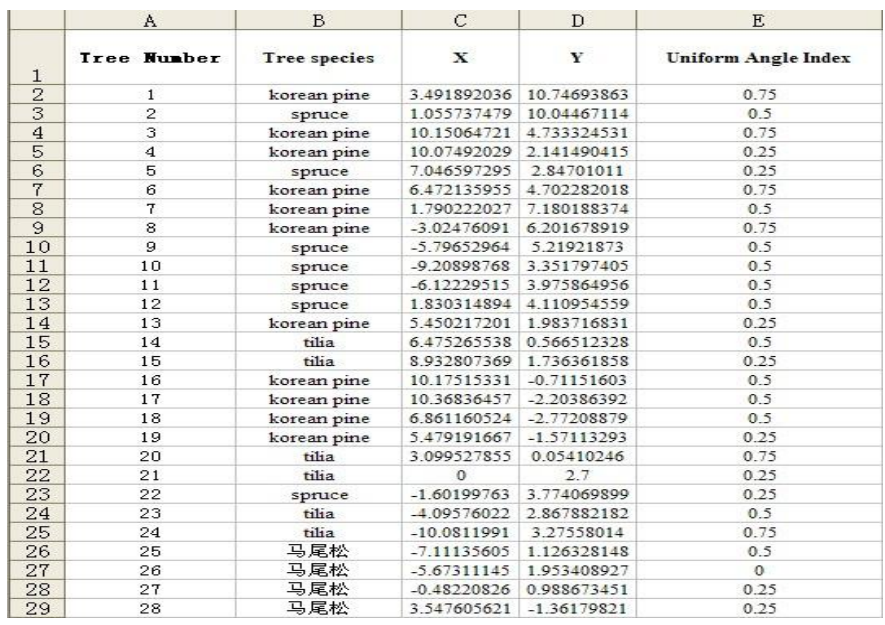

Figure4. The sheets of Analysis Tools of Uniform Angle Index

H 1 , M Prinitive data/Nlean value of UaI/

Figure5. The names of analysis tools of uniform angle index

\subsubsection{The design of user interface}

Firstly, a commandbar was defined and established, then controls commandbarpopup were added with the method "add ( )". Finally, dropdown menus were acquired by applying "add ( )" method of controls commandbarpopup to msocontrolbutton. The system includes two dropdown menus: "Data management" and "Calculating Uniform Angle Index" (Figure 3). The codes were as flow:

Public Sub addpopupbar()

Set cmbnewbar $=$ CommandBars.Add(Name:="Analysis Tools of Uniform Angle Index ")

With cmbnewbar

Set mbtn $=$.Controls.Add(msoControlPopup)

With mbtn

.Caption $=$ "Data Management"

Set ctlbtn $=$.Controls. Add

With ctlbtn

.Caption $=$ "Open File"

.OnAction = "Open File"

End With

Set ct 1 btn $=$.Controls. Add

With ct1btn

.Caption $=$ "Save File"
.OnAction = "Save File"

End With

Set $\operatorname{ct} 1 \mathrm{btn}=$.Controls. Add

With ct1btn

.Caption $=$ "Delete Data"

.OnAction = "Delete Data"

End With

End With

.Visible $=$ True

Set mbtn $1=$.Controls.Add(msoControlPopup)

With mbtn1

.Caption $=$ "Calculating Uniform Angle Index"

Set ctlbtn $1=$.Controls.Add

With ctlbtn1

.Caption = "Calculating Uniform Angle Index"

.OnAction = "Calculating Uniform Angle Index"

End With

.Visible $=$ True

End With

.Visible $=$ True

End With

End Sub

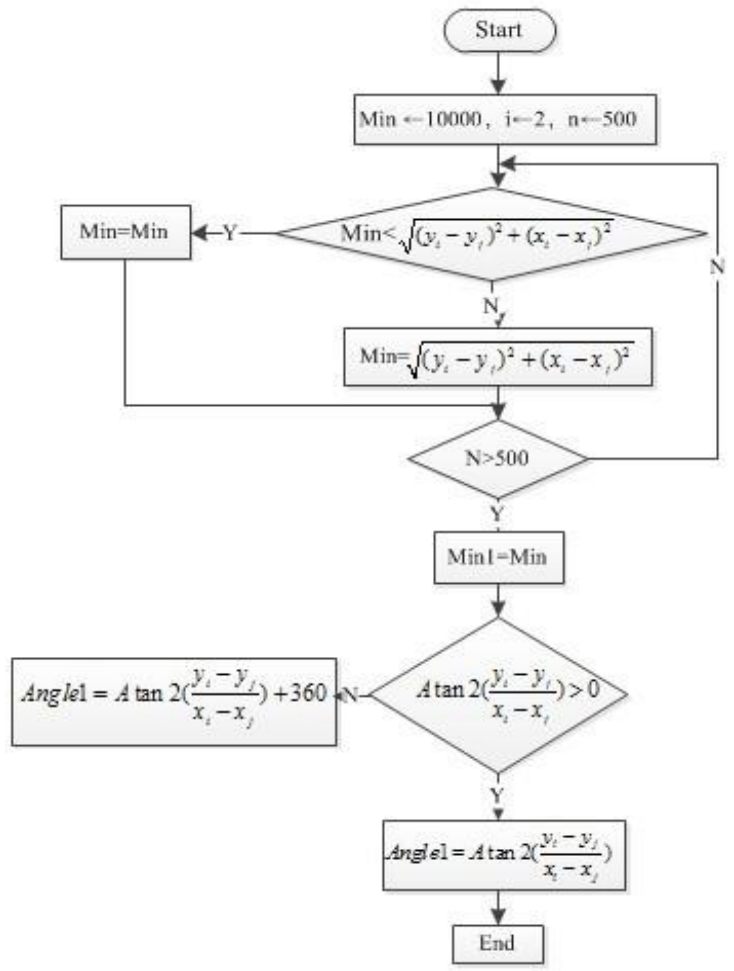

Figure6a. Flow chart of analysis tools of uniform angle index

\subsubsection{The design of main modules}

The main modules were divided into there part: the module of computing four closest distances, the module of calculating the standards angles and the module of counting uniform angle index of each tree and the mean value of uniform angle index of stand in forest.

The module of computing four closest distances was designed to compute the four closest distances from four closest neighboring trees to reference tree with the function of minimum value transmission. The main codes calculating the first closest distances were as follow: 
Worksheets("Primitive data "). Activate

For $x i=2$ To RowNumber

$\mathrm{t}=1000$

For yj $=2$ To RowNumber

If yj $<>$ xi Then

If $\mathrm{t}<(($ Cells $(x i, 3)$.Value - Cells(yj,

3).Value $\left.)^{\wedge} 2+(\text { Cells }(x i, 4) \cdot \text { Value }- \text { Cells }(y j, 4) \cdot \text { Value })^{\wedge} 2\right)^{\wedge}$

0.5 And $\mathrm{t}>0$ Then

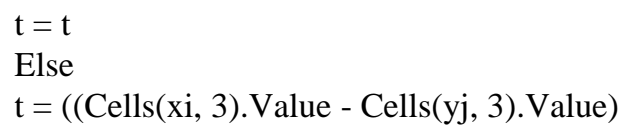

$\mathrm{t}=1000$

Next xi

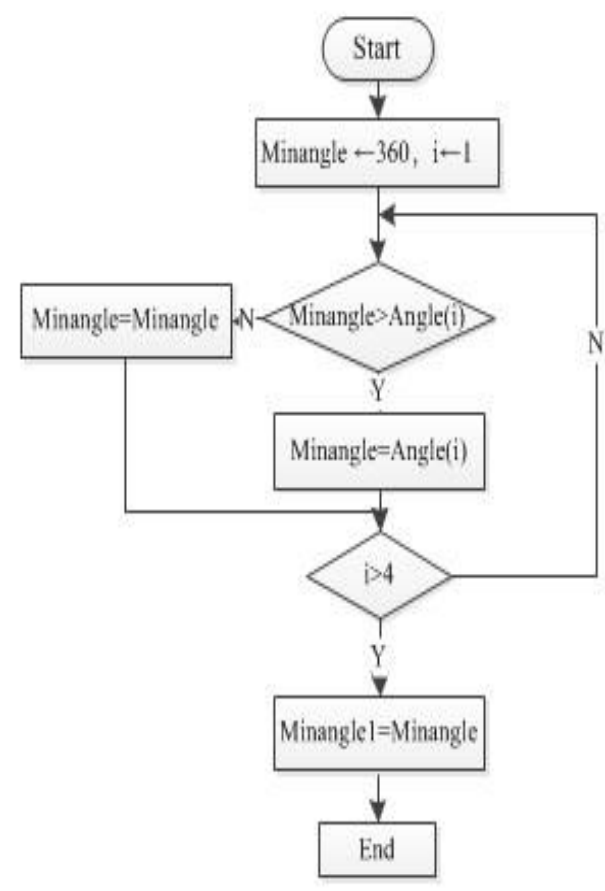

Figure6b. Flow chart of analysis tools of uniform angle index

The integrated module of calculating the standards angle involved process of computing the angles $(\theta)$, ranking the angles and counting the standards angle. The main codes were as follow:

For yj = 2 To RowNumber

$$
\text { If yj }<>\text { xi Then }
$$

If $\left((\text { Cells }(x i, 3) \text {.Value - Cells }(y j, 3) . \text { Value })^{\wedge} 2+\right.$

$\left.(\text { Cells }(x i, 4) . V a l u e-C e l l s(y j, 4) . V a l u e)^{\wedge} 2\right)^{\wedge} 0.5=\mathrm{t} 1$ Then If Cells $(y j, 3)$.Value $=$ Cells $(x i, 3)$.Value

Then

4).Value Then

$$
\text { If Cells(yj, 4).Value > Cells(xi, }
$$

$$
\text { angle } 1=270
$$$$
\text { Else }
$$$$
\text { angle } 1=90
$$$$
\text { End If }
$$

Else
hudu=Application.WorksheetFunction.Atan2((Cells(yj,3).Valu e - Cells(xi, 3).Value), (Cells(yj, 4).Value - Cells(xi, 4).Value)) angle $=$

Application.WorksheetFunction.Degrees(hudu)

If angle $>0$ Then

angle $1=$ angle

Else

angle $1=$ angle +360

End If

End If

\section{End If}

Next yj

$$
\begin{gathered}
\text { angle }(1)=\text { angle } 1 \\
\text { angle }(2)=\text { angle } 2 \\
\text { angle }(3)=\text { angle3 } \\
\text { angle }(4)=\text { angle } 4 \\
\text { minangle = } 360
\end{gathered}
$$

For $\mathrm{i}=1$ To 4

If angle (i) < minangle Then

End If minangle $=$ angle $(\mathrm{i})$

Next i

Minangle $(1)=$ minangle

For $\mathrm{j}=1$ to 3

If Minangle( $j+1)-M i n a n g l e(j)<180 \quad$ then includedangle $(\mathrm{j})=\operatorname{Minangle}(\mathrm{j}+1)$-Minangle $(\mathrm{j})$

Else

includedangle $(j)=360-(\operatorname{Minangle}(j+1)$ -

Minangle(j))

End If

If Minangle(4)-Minangle(1) $<180$ then

includedangle (4) = Minangle(4)-Minangle(1)

Else

Minangle(1))

includedangle (4)=360-( Minangle(4)-

Next j

The module of calculating uniform angle index contained two sub-modules: counting uniform angle index of each tree and calculating the mean value of uniform angle index of stand in forest. The main codes were as follow:

$$
\text { For } \mathrm{i}=1 \text { To } 4
$$

If jiajiaoji(i) $>72$ Or jiajiaoji(i) $=72$ Then

$$
\mathrm{n}=\mathrm{n}+1
$$

End If

Next $\mathrm{i}$

If $n=4$ Then

$$
\text { wi }=0
$$

End If

If $\mathrm{n}=3$ Then

$$
\text { wi }=0.25
$$

End If

If $\mathrm{n}=2$ Then

wi $=0.5$

End If

If $\mathrm{n}=1$ Then

wi $=0.75$

End If

If $\mathrm{n}=0$ Then

wi $=1$ 


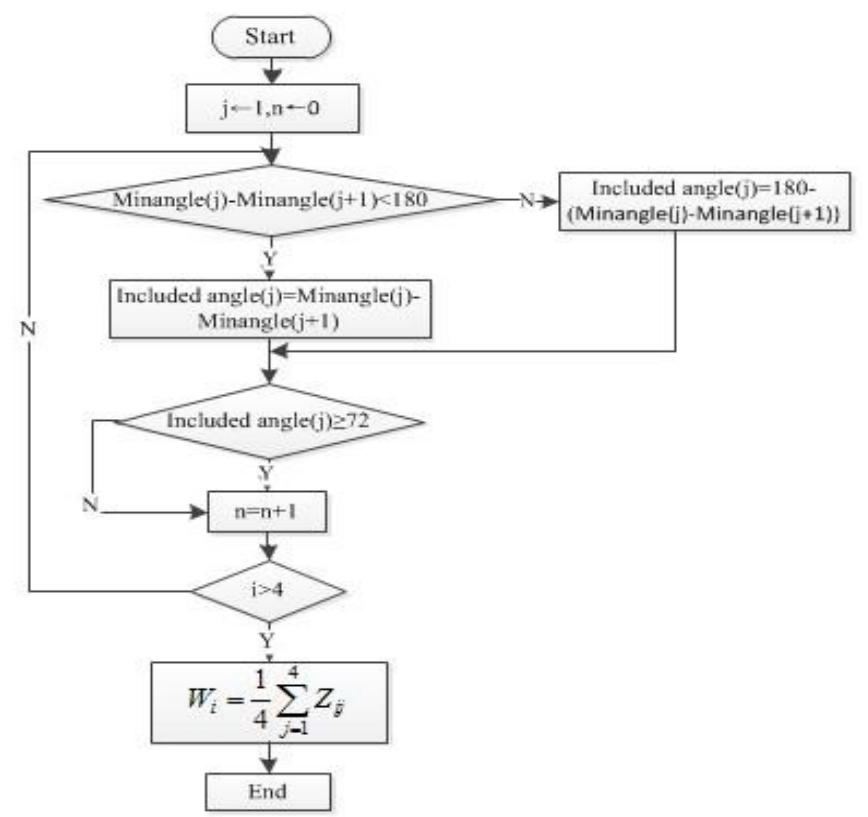

Figure6c. Flow chart of analysis tools of uniform angle index

$$
\begin{aligned}
& \text { End If } \\
& \text { Next yj } \\
& \text { swi }=\text { swi }+ \text { wi } \\
& \text { Cells (xi, 5). Value }=\text { wi } \\
& \text { For } x i=2 \text { To RowNumber } \\
& \text { If }\left((\text { Cells }(x i, 3) . \text { Value })^{\wedge} 2+(\text { Cells }(x i, 3) . \text { Value })\right. \\
& \wedge 2)^{\wedge} 0.5<=10.29 \text { Then } \\
& \text { If Cells(xi, 5).Value }=0 \text { Then } \\
& \mathrm{n} 0=\mathrm{n} 0+1 \\
& \text { End If } \\
& \text { If Cells(xi, 5).Value }=0.25 \text { Then } \\
& \mathrm{n} 025=\mathrm{n} 025+1 \\
& \text { End If } \\
& \text { If Cells }(\mathrm{xi}, 5) \text {.Value }=0.5 \text { Then } \\
& \mathrm{n} 05=\mathrm{n} 05+1 \\
& \text { End If } \\
& \text { If Cells(xi, 5).Value }=0.75 \text { Then } \\
& \mathrm{n} 075=\mathrm{n} 075+1 \\
& \mathrm{n} 100=\mathrm{n} 100+1
\end{aligned}
$$

Next xi

\section{CONCLUSION}

The Analysis Tools of Uniform Angle Index has been developed by utilizing Excel VBA programming language.

Excel VBA programming language has many merits. Firstly, development environment and operating environment of Excel VBA are EXCEL.
Hence, the programming language can recall expediently and directly data in Excel. Moreover, due to no requirement of development of database module, workload of programming is greatly reduced. Furthermore, Excel workbook itself has very powerful statistical analysis and charting function, which can be implemented by VBA. Finally, it is easy to learn.

\section{ACKNOWLEDGEMENTS}

The paper was supported by Fundamental Research Funds for the Central Universities (LGYB201401).

\section{REFERENCES}

[1] Hui G.Y., Klaus von Gadow, Hu Y.B. \& Xu H. 2007. Structure-based forest management. Beijing: Forestry Publishing House.

[2] HUI G.Y., Klaus von Gadow \& HU Y.B., et al. 2004. Characterizing forest spatial distribution pattern with the mean value of uniform angle index. Acta Ecologica Sinica 24(6):1225-1229.

[3] HUI G.Y. 1999. the neighbourhood pattern-a new structure parameter for describing distribution of forest tree position. Scientia Silvae Sinicae 35 (1):37-42.

[4] Tang Y., Zhao K. \& Xu W.B. 2011. Development of VBA for pump performance experimental data based on Excel. Paiguan Ji xie Xuebao 29(2):123-126.

[5] Wang Y.M. 2009.The calculation of urban gini coefficient in China by excel VBA. In Proceeding(s) of the 2009 ETP/IITA World Congress in Applied Computing, Computer Science, and Computer Engineering.

[6] Zhao H.Y. \& Lu Y.C., et al. 2011.Design and development of supporting tool for target-tree-operation management. In Proceeding(s) of the 2011 International Conference on Computer and Communication Devices, $\mathrm{Li}$ Yang, pp: VI40-VI43.

[7] Zhao H.Y. \& Kang X.G., et al. 2011.A kind of algorithm estimating parameters of unitary non-linearity regression model. In Proceeding(s) of the 2011 3rd International Conference on Machine Learning and Computing, Dr.Steve Thatcher, pp: V317- V319.

[8] Zhao H.Y. \& Kang X.G., et al. 2011.Design and development of a tool for fitting Weibull distribution function based on EXCEL VBA. In Proceeding(s) of the 2011 International Conference on Information and Computer Applications, Li Yuting, pp: 396-399.

[9] Zhao H.Y. \& Kang X.G., Hua Y., et al. 2013. Design and Development of Sapling Growth Simulation System. Journal of Converagence Information Technology, 5 (5):224-231.

[10] Zhou J.B. \& Li X.H. 2006. GeoPlot: An Excel VBA program for geochemical data plotting Computers \& Geosciences 32(4): 554-560. 